\title{
A New Mathematical Modeling with Optimal Control Strategy for the Dynamics of Population of Diabetics and Its Complications with Effect of Behavioral Factors
}

\author{
Abdelfatah Kouidere $\mathbb{D}^{1},{ }^{1}$ Abderrahim Labzai $\mathbb{D},{ }^{1}$ Hanane Ferjouchia, ${ }^{1}$ Omar Balatif $\mathbb{D},{ }^{2}$ \\ and Mostafa Rachik $(\mathbb{D})^{1}$ \\ ${ }^{1}$ Laboratory of Analysis Modeling and Simulation, Department of Mathematics and Computer Science, Faculty of Sciences Ben M'Sik, \\ Hassan II University, Sidi Othman, Casablanca, Morocco \\ ${ }^{2}$ Laboratory of Dynamical Systems, Mathematical Engineering Team (INMA), Department of Mathematics, Faculty of Sciences \\ El Jadida, Chouaib Doukkali University, El Jadida, Morocco
}

Correspondence should be addressed to Abdelfatah Kouidere; kouidere89@gmail.com

Received 3 March 2020; Revised 23 April 2020; Accepted 16 May 2020; Published 4 June 2020

Academic Editor: Bruno Carpentieri

Copyright (C) 2020 Abdelfatah Kouidere et al. This is an open access article distributed under the Creative Commons Attribution License, which permits unrestricted use, distribution, and reproduction in any medium, provided the original work is properly cited.

We propose an optimal control strategy by conducting awareness campaigns for diabetics about the severity of complications of diabetes and the negative impact of an unbalanced lifestyle and the surrounding environment, as well as treatment and psychological follow-up. Pontryagin's maximum principle is used to characterize the optimal controls, and the optimality system is solved by an iterative method. Finally, some numerical simulations are performed to verify the theoretical analysis using MATLAB.

\section{Introduction}

Nowadays, diabetes is a chronic disease with a huge burden affecting individuals. According to the World Health Organisation (WHO) [1], diabetes is a disorder characterized by the presence of problems in the insulin hormone, which naturally results from the pancreas to help the body use glucose and fat and store some of them. According to the American Diabetes Association (ADA) [2], diabetes mellitus is a group of metabolic diseases characterized by hyperglycemia resulting from defects in insulin secretion, insulin action, or both. It is known that the proper level of glucose in the blood after fasting eight hours should be less than $108 \mathrm{mg} / \mathrm{dl}$, while the borderline is $126 \mathrm{mg} / \mathrm{dl}$. If a person's blood glucose level is $126 \mathrm{mg} / \mathrm{dL}$ and above, in two or more tests, then that person is diagnosed with diabetes. Diabetes is divided into several different types; some more prevalent than others. The most common type of diabetes in the general population is type 2 diabetes, and type 1 diabetes is more common in children, and gestational diabetes is a form of diabetes that can occur during pregnancy. According to the latest statistics from the International Diabetes Federation (IDF) and as reported in the 9th edition of the Atlas Diabetes 2017 [3], diabetes is a constantly growing disease. There are more than 370 million people with diabetes worldwide $(8.5 \%$ of the adult population) and about 463 million people in prediabetes $(6.5 \%$ of the adult population), and more than 625 million are expected to be affected by to 2045 .

Today, all countries of the world suffer from the high number of people with diabetes, which is increasing and expanding on the extreme level. When it is not treated well, all types of diabetes can lead to complications in many parts of the body, leading to an early death. When a diabetic knows how to control the level of glucose in the blood, this awareness plays a key role in reducing the serious complications of diabetes. According to IDF statistics, diabetes has serious and varied complications. For example, the risk of cardiovascular disease. Moreover, more than a third of diabetics have 


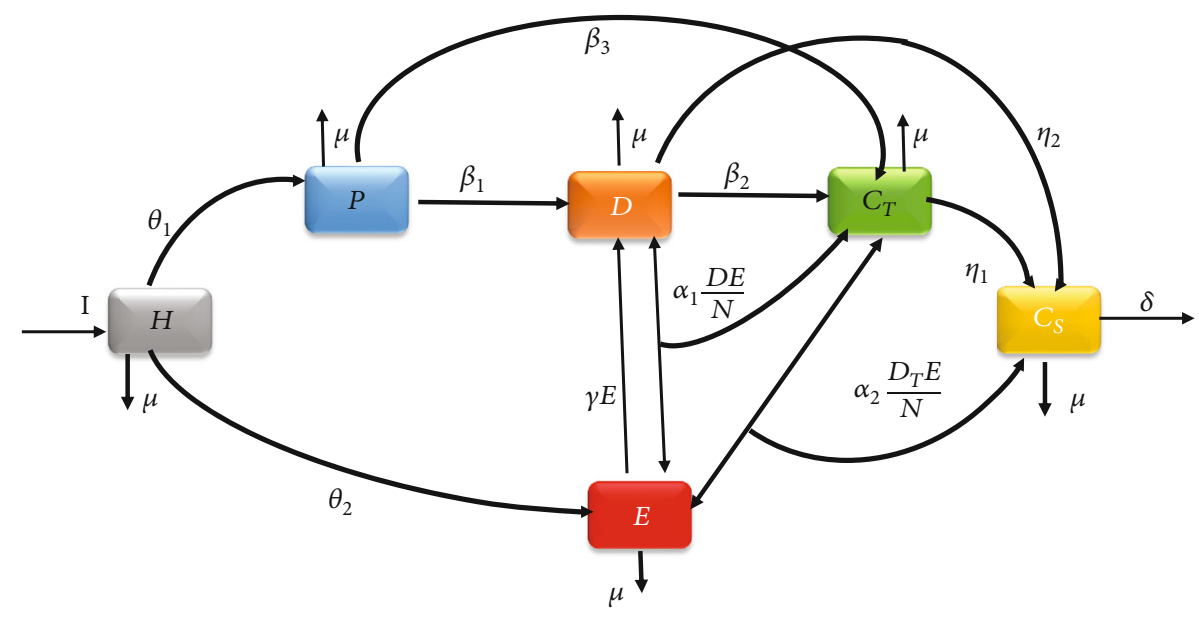

Figure 1: The dynamics of a population of diabetics.

retinopathy which is the main cause of vision loss, in addition to the risk of kidney disease. In addition, the complications of diabetes are multiple and different depending on the degree of severity; there are complications that can be treated and others that have reached critical stages with which treatment is not beneficial. According to ADA [20], diabetes has economic and social burdens on the individual and society.

During the last decade, large mathematical models on diabetes have been developed to simulate, analyse, and understand the dynamics of a population of diabetics. In a related research work, Boutayeb and Chetouani [4] and Derouich et al. [5] introduced a mathematical model for the dynamics of the population of diabetes. And Kouidere et al. [6] proposed a discrete mathematical model highlighting the impact of living environment. Also, many researches have focused on this topic and other related topics ([7-12]).

As we said earlier, diabetes has many complications, and the nature of these complications are two types: treatable ones and those that have reached a critical stage which is impossible to cure completely.

According to IDF [3], diabetes is influenced by a complex interaction of behavioural, genetic, and socioeconomic factors; many of which are outside our individual control.

To achieve this objective, we considered a compartment model that describes the dynamics of a population of diabetics that is divided into six compartments, i.e., the healthy people $(H)$, the prediabetics through genetic factors $(P)$, the prediabetics through the negative effect of behavioral factors on diabetics patients and others $(E)$, and $(D)$ diabetics without complications are those who control blood sugar through diet and exercise before it is too late, and we divided diabetics with complications into two parts: $C_{T}$ diabetics with treatable complication and $C_{S}$ diabetics with serious complications.

We noticed that most of researchers about diabetes and its complications focused on continuous and discrete time models and described by differential equations. Recently, more and more attention has been paid to study the control optimal (see [13-21] and the references mentioned there).

In this paper, in Section 2, we represent a $H P E D C_{T} C_{S}$ mathematical model that describes the dynamic of a popula- tion of diabetics. In Section 3, we presented an optimal control problem for the proposed model, where we gave some results concerning the existence and positivity of the optimal control and we characterized the optimal controls used Pontryagin's maximum principle. Numerical simulations through MATLAB are given in Section 4. Finally, we conclude the paper in Section 5.

\section{A Mathematical Model}

We consider a mathematical model $\mathrm{HPEDC}_{T} C_{S}$ that describes the dynamics of a population of diabetics. We divide the population denoted by $N$ into six compartments.

2.1. Description of the Model. The graphical representation of the proposed model is shown in Figure 1.

The compartment $(H)$ are healthy people; the compartment $H$ is increased by $I$ (which is the recruitment rate of healthy people), and this compartment $H$ is decreased by $\theta_{1}$ $H(t)$ (the number of prediabetic people through genetic factor) and also decreased by $\theta_{2} H(t)$ (the number of prediabetics through the negative effect of behavioral factors) and decreased by the amount $\mu$ (natural mortality).

$$
\frac{d H(t)}{d t}=I-\left(\mu+\theta_{1}+\theta_{2}\right) H(t)
$$

The compartment $(P)$ are people who are likely to have diabetes through genetic factors. The compartment $P$ is increased by $\theta_{1} H(t)$. This compartment $P$ is decreased by the amount $\mu$ (natural mortality) and also decreased by $\beta_{1} P(t)$ (the probability of developing diabetes) and by $\beta_{3} C_{T}(t)$ (the probability of developing diabetes at stage of complications).

$$
\frac{d P(t)}{d t}=\theta_{1} H(t)-\left(\mu+\beta_{1}+\beta_{3}\right) P(t) .
$$


Compartment $(E)$ are people who are likely to have diabetes through the negative effect of lifestyle or psychological problem factors and others (these are people at risk of developing diabetes, such as those who are obese, overweight, gestational diabetes, or due to family and work problems, in addition to the person without diabetes).The compartment $E$ increased by $\theta_{2} H(t)$ and decreased by $\gamma E(t)$ (patients who become diabetics without complications because of the negative effect of lifestyle) and also decreased by $\mu$ (natural mortality).

$$
\frac{d E(t)}{d t}=\theta_{2} H(t)-(\mu+\gamma) E(t)
$$

Compartment $(D)$ is the number of diabetics without complications. The compartment $D(t)$ increased by the amount $\beta_{1} P(t)$ and by the amount $\gamma E(t)$. This compartment $D$ is decreasing by $\mu$ (natural mortality) and $\alpha_{1}$ (the rate of negative impact of ordinary people on diabetics without complications through improper nutrition, or practical or family social problems) and also decreased by $\beta_{2} D(t)$ (the probability of a diabetic person developing a complication) and also decreased by $\eta_{2} D(t)$ (the number of diabetics whose serious complications because of a sudden shock).

$$
\begin{aligned}
\frac{d D(t)}{d t}= & \beta_{1} P(t)+\gamma E(t)-\alpha_{1} \frac{D(t) E(t)}{N} \\
& -\left(\mu+\beta_{2}+\eta_{2}\right) D(t) .
\end{aligned}
$$

Compartment $\left(\mathbf{C}_{T}\right)$ is the number of diabetics with treatable complications. The compartment $\mathbf{C}_{T}$ increased by $\beta_{3} P(t)$ and by $\beta_{2} D(t)$ and also increased by $\alpha_{1}$ and and decreased by $\alpha_{2}$ (the rate negative impact of ordinary people on diabetics with treatable complications through improper nutrition, or practical or family social problems) and decreased by $\eta_{1} \mathbf{C}_{T}(t)$ (the number of people developing of diabetics with complications can be treated to serious complications) and also by $\mu$ (natural mortality)

$$
\begin{aligned}
\frac{d \mathbf{C}_{T}(t)}{d t}= & \beta_{3} P(t)+\beta_{2} D(t)+\alpha_{1} \frac{D(t) E(t)}{N} \\
& -\alpha_{2} \frac{\mathbf{C}_{T}(t) E(t)}{N}-\left(\mu+\eta_{1}\right) C_{T}(t) .
\end{aligned}
$$

Compartment $\left(\mathbf{C}_{S}\right)$ is the number of diabetics with serious complications, they are the diabetics who have serious complications such as retinopathy or renal failure. The compartment $\mathbf{C}_{S}=\mathbf{C}_{S}(t)$ increased by $\eta_{2} D(t)$ and also by $\eta_{1} \mathbf{C}_{T}(t)$ and by $\alpha_{2}$. This compartment $\mathbf{C}_{S}$ decreased by $\delta$ (mortality rate due to complications) and also by $\mu$ (natural mortality).

$$
\frac{d \mathbf{C}_{S}(t)}{d t}=\eta_{2} D(t)+\eta_{1} \mathbf{C}_{T}(t)+\alpha_{2} \frac{\mathbf{C}_{T}(t) E(t)}{N}-(\mu+\delta) C_{S}(t) .
$$

Hence, we present the diabetic model by the following system of differential equations:

$$
\left\{\begin{array}{l}
\frac{d H(t)}{d t}=I-\left(\mu+\theta_{1}+\theta_{2}\right) H(t), \\
\frac{d P(t)}{d t}=\theta_{1} H(t)-\left(\mu+\beta_{1}+\beta_{3}\right) P(t), \\
\frac{d E(t)}{d t}=\theta_{2} H(t)-(\mu+\gamma) E(t), \\
\frac{d D(t)}{d t}=\beta_{1} P(t)+\gamma E(t)-\alpha_{1} \frac{D(t) E(t)}{N}-\left(\mu+\beta_{2}+\eta_{2}\right) D(t), \\
\frac{d \mathbf{C}_{T}(t)}{d t}=\beta_{3} P(t)+\beta_{2} D(t)+\alpha_{1} \frac{D(t) E(t)}{N}-\alpha_{2} \frac{\mathbf{C}_{T}(t) E(t)}{N}-\left(\mu+\eta_{1}\right) C_{T}(t), \\
\frac{d \mathbf{C}_{S}(t)}{d t}=\eta_{2} D(t)+\eta_{1} \mathbf{C}_{T}(t)+\alpha_{2} \frac{\mathbf{C}_{T}(t) E(t)}{N}-(\mu+\delta) C_{S}(t),
\end{array}\right.
$$

with $H(0) \geq 0, P(0) \geq 0, E(0) \geq 0, D(0) \geq 0, \mathbf{C}_{T}(0) \geq 0$, and $\mathrm{C}_{S}(0) \geq 0$.

\subsection{Positivity of Solutions}

Theorem 1. If $H(0) \geq 0, P(0) \geq 0, E(0) \geq 0, D(0) \geq 0, \boldsymbol{C}_{T}(0)$ $\geq 0$, and $C_{S}(0)>0$, the solutions $H(t), P(t), E(t), D(t)$, $\boldsymbol{C}_{T}(t)$, and $\boldsymbol{C}_{S}(t)$ of system (7) are positive for all $t \geq 0$.
Proof. It follows from the first equation of system (7) that

$$
\begin{gathered}
\frac{d H(t)}{d t}=I-\left(\mu+\theta_{1}+\theta_{2}\right) H(t) \geq-\left(\mu+\theta_{1}+\theta_{2}\right) H(t) \\
\frac{d H(t)}{d t}+\left(\mu+\theta_{1}+\theta_{2}\right) H(t) \geq 0
\end{gathered}
$$


Both sides in the last inequality are multiplied with exp $\left(\left(\mu+\theta_{1}+\theta_{2}\right) t\right)$.

We obtain

$$
\begin{aligned}
& \exp \left(\left(\mu+\theta_{1}+\theta_{2}\right) t\right) \cdot \frac{d H(t)}{d t} \\
& \quad+\left(\mu+\theta_{1}+\theta_{2}\right) \exp \left(\left(\mu+\theta_{1}+\theta_{2}\right) t\right) \cdot H(t) \geq 0,
\end{aligned}
$$

then

$$
\frac{d}{d t}\left(\exp \left(\left(\mu+\theta_{1}+\theta_{2}\right) t\right) \cdot H(t)\right) \geq 0
$$

Integrating this inequality from 0 to $t$ gives

$$
\int_{0}^{t} \frac{d}{d s}\left(\exp \left(\left(\mu+\theta_{1}+\theta_{2}\right) s\right) \cdot H(s)\right) d s \geq 0
$$

then

$$
H(t) \geq H(0) \exp \left(\left(\mu+\theta_{1}+\theta_{2}\right) t\right) \Rightarrow H(t)>0 .
$$

Similarly, we prove that $P(t) \geq 0, E(t) \geq 0, D(t) \geq 0, \mathbf{C}_{T}$ $(t) \geq 0$, and $\mathbf{C}_{S}(t) \geq 0$.

\subsubsection{Boundedness of the Solutions}

Theorem 2. The set $\Omega=\left\{\left(H, P, E, D, C_{T}, C_{S}\right) \in \mathbb{R}^{5} / 0 \leq H+\right.$ $\left.P+E+D+C_{T}+C_{S} \leq I / \mu\right\}$ is positively invariant under system (7) with initial conditions $H(0) \geq 0, P(0) \geq 0, E(0) \geq 0$, $D(0) \geq 0, \boldsymbol{C}_{T}(0) \geq 0$, and $\boldsymbol{C}_{S}(0)>0$.

Proof. By adding the equations of system (7), we obtain

$$
\frac{d N}{d t}=I-\mu N-\delta C_{S} \leq I-\mu N \Rightarrow N(t) \leq \frac{I}{\mu}+N(0) e^{-\mu t},
$$

where $N(0)$ represents the initial values of the total population.

Thus, $\lim _{t \rightarrow \infty} \sup N(t)=(I / \mu)$. It implies that the region $\Omega$ is a positively invariant set for system (7). So, we only need to consider the dynamics of the system on the set $\Omega$.

\subsubsection{Existence of Solutions}

Theorem 3. The system (7) that satisfies a given initial condition $\left(H(0), P(0), E(0), D(0), C_{T}(0)\right.$, and $\left.C_{S}(0)\right)$ has a unique solution.

Proof. Let

$$
X=\left(\begin{array}{c}
S(t) \\
P(t) \\
E(t) \\
D(t) \\
\mathrm{C}_{T}(t) \\
\mathrm{C}_{S}(t)
\end{array}\right),
$$

$$
\varphi(X)=\left(\begin{array}{c}
d H(t) / d t \\
d P(t) / d t \\
d E(t) / d t \\
d D(t) / d t \\
d \mathbf{C}_{T}(t) / d t \\
d \mathbf{C}_{S}(t) / d t
\end{array}\right),
$$

so the system (7) can be rewritten in the following form:

$$
\varphi(\dot{X})=X=A X+B(X)
$$

where

$$
\begin{aligned}
A & =\left(\begin{array}{cccccc}
-\left(\mu+\theta_{1}+\theta_{2}\right) & 0 & 0 & 0 & 0 & 0 \\
\theta_{1} & -\left(\mu+\beta_{1}+\beta_{3}\right) & 0 & 0 & 0 & 0 \\
\theta_{2} & 0 & -\mu+\gamma & 0 & 0 & 0 \\
0 & \beta_{1} & \gamma & -\left(\mu+\beta_{2}+\eta_{2}\right) & 0 & 0 \\
0 & \beta_{3} & 0 & \beta_{2} & -\left(\mu+\eta_{1}\right) & 0 \\
0 & 0 & 0 & \eta_{2} & \eta_{1} & -(\mu+\delta)
\end{array}\right), \\
B(X) & =\left(\begin{array}{c}
\frac{I}{2} \\
-\alpha_{1} \frac{D(t) E(t)}{N} \\
\alpha_{1} \frac{D(t) E(t)}{N}-\alpha_{2} \frac{\mathbf{C}_{T}(t) E(t)}{N} \\
\alpha_{2} \frac{\mathbf{C}_{T}(t) E(t)}{N}
\end{array}\right) .
\end{aligned}
$$


The second term on the right-hand side of (15) satisfies

$$
\begin{aligned}
& \left|B\left(X_{1}\right)-B\left(X_{2}\right)\right| \leq M\left(\left|D_{1}(t)-D_{2}(t)\right|+\left|\mathbf{C}_{T .1}(t)-\mathbf{C}_{T .2}(t)\right|\right), \\
& \left|B\left(X_{1}\right)-B\left(X_{2}\right)\right| \leq M \cdot\left\|X_{1}-X_{2}\right\|
\end{aligned}
$$

where $M=2(I / \mu)\left(\left|\alpha_{1} / N\right|+\left|\alpha_{2} / N\right| ;\left|\alpha_{1} / N\right|+\left|\alpha_{2} / N\right|\right)$, then

$$
\left\|\varphi\left(X_{1}\right)-\varphi\left(X_{2}\right)\right\| \leq V \cdot\left\|X_{1}-X_{2}\right\|
$$

where $V=\max (M,\|A\|)<\infty$.

Thus, it follows that the function $\varphi$ is uniformly Lipschitz continuous, and the restriction on $H(t) \geq 0, P(t) \geq 0, E(t) \geq$ $0, D(t) \geq 0, \mathbf{C}_{T}(t) \geq 0$, and $\mathbf{C}_{S}(t) \geq 0$, we see that a solution of the system (7) exists [22].

\section{Formulation of the Model}

Our objective in this proposed strategy of control is to minimize the number of people evolving from the stage of prediabetes to the stages of diabetes without complications and to diabetes with treatable complications. In this model, we include four controls $u_{1}(t), u_{2}(t), u_{3}(t)$, and $u_{4}(t)$ for $t \in[0$, $\left.T_{f}\right]$. The first control we note $u_{1}$ represented treatment that will work to treat diabetic patients with treatable complications. The second control $u_{2}$ represented the awareness program through media and education, by raising awareness and awareness of the seriousness of the negative impact of behavioral factors on diabetes. The third control $u_{3}$ represented treatment and education, by treating complications, with sensitivity to the negative effect of behavioral factors on diabetic patients with complications. The fourth control $u_{4}$ represented the awareness program, through raising awareness on nondiabetics against the negative impact of behavioral, economic, and social factors that lead to diabetes at time $t$.

So the controlled mathematical system is given by the following system of differential equations:

$$
\left\{\begin{array}{l}
\frac{d H(t)}{d t}=I-\left(\mu+\theta_{1}+\theta_{2}\right) H(t) \\
\frac{d P(t)}{d t}=\theta_{1} H(t)-\left(\mu+\beta_{1}+\beta_{3}\right) P(t) \\
\frac{d E(t)}{d t}=\theta_{2} H(t)-\mu E(t)-\gamma\left(1-u_{4}(t)\right) E(t) \\
\frac{d D(t)}{d t}=\beta_{1} P(t)+\gamma\left(1-u_{4}(t)\right) E(t)-\alpha_{1}\left(1-u_{2}(t)\right) \frac{D(t) E(t)}{N}-\left(\mu+\beta_{2}+\eta_{2}\right) D(t)+u_{1}(t) C_{T}(t) \\
\frac{d \mathbf{C}_{T}(t)}{d t}=\beta_{3} P(t)+\beta_{2} D(t)+\alpha_{1}\left(1-u_{2}(t)\right) \frac{D(t) E(t)}{N}-\alpha_{2}\left(1-u_{3}(t)\right) \frac{\mathbf{C}_{T}(t) E(t)}{N}-\left(\mu+\eta_{1}+u_{1}(t)\right) C_{T}(t) \\
\frac{d \mathbf{C}_{S}(t)}{d t}=\eta_{2} D(t)+\eta_{1} \mathbf{C}_{T}(t)+\alpha_{2}\left(1-u_{3}(t)\right) \frac{\mathbf{C}_{T}(t) E(t)}{N}-(\mu+\delta) C_{S}(t) .
\end{array}\right.
$$

The problem is to minimize the objective functional

$$
\begin{aligned}
J\left(u_{1}, u_{2}, u_{3}, u_{4}\right)= & C_{T}\left(T_{f}\right)-D\left(T_{f}\right)-E\left(T_{f}\right) \\
& +\int_{0}^{T_{f}} C_{T}(t)-D(t)-E(t)+\frac{A}{2} u_{1}^{2}(t) \\
& \left.+\frac{B}{2} u_{2}^{2}(t)+\frac{F}{2} u_{3}^{2}(t)+\frac{G}{2} u_{4}^{2}(t)\right] d t
\end{aligned}
$$

where $A>0, B>0, F>0$, and $G>0$ are the cost coefficients; they are selected to weigh the relative importance of $u_{1}(t)$, $u_{2}(t), u_{3}(t)$, and $u_{4}(t)$ at time $t$, and $T_{f}$ is the final time.

In other words, we seek the optimal controls $u_{1}^{*}(t)$, $u_{2}^{*}(t), u_{3}^{*}(t)$, and $u_{4}^{*}(t)$ such that

$$
J\left(u_{1}^{*}, u_{2}^{*}, u_{3}^{*}, u_{4}^{*}\right)=\min _{u_{1}, u_{2}, u_{3}, u_{4} \in U} J\left(u_{1}, u_{2}, u_{3}, u_{4}\right),
$$

where $U$ is the set of admissible controls defined by

$$
\begin{aligned}
U & =\left\{\left(u_{1}, u_{2}, u_{3}, u_{4}\right) / 0 \leq u_{1 \min } \leq u_{1}(t) \leq u_{1 \max }\right. \\
& \leq 1,0 \leq u_{2 \min } \leq u_{2}(t) \leq u_{2 \max } \leq 1,0 \leq u_{3 \min } \\
& \leq u_{3}(t) \leq u_{3 \max } \leq 1,0 \leq u_{4 \min } \leq u_{4}(t) \leq u_{4 \max } \\
& \left.\leq 1 / t \in\left[0, T_{f}\right]\right\}
\end{aligned}
$$

\section{The Optimal Control: Existence and Characterization}

We first show the existence of solutions of the system (18), thereafter, we will prove the existence of optimal control.

\subsection{Existence of an Optimal Control}

Theorem 4. Consider the control problem with system (18). There exists an optimal control $\left(u_{1}^{*}, u_{2}^{*}, u_{3}^{*}, u_{4}^{*}\right) \in U^{4}$ such that

$$
J\left(u_{1}^{*}, u_{2}^{*}, u_{3}^{*}, u_{4}^{*}\right)=\min _{u_{1}, u_{2}, u_{3}, u_{4} \in U} J\left(u_{1}, u_{2}, u_{3}, u_{4}\right)
$$


Proof. The existence of the optimal control can be obtained using a result by Fleming and Rishel [23], checking the following steps:

(i) It follows that the set of controls and corresponding state variables is not empty. we will use a simplified version of an existence result ([24], Theorem 7.1.1)

(ii) $J\left(u_{1}, u_{2}, u_{3}, u_{4}\right)$ is convex in $U$

(iii) The control space $U=\left\{\left(u_{1}, u_{2}, u_{3}, u_{4} / u_{1}, u_{2}, u_{3}, u_{4}\right)\right.$ is measurable, $0 \leq u_{1 \min } \leq u_{1}(t) \leq u_{1 \max } \leq 1,0 \leq$ $u_{2 \min } \leq u_{2}(t) \leq u_{2 \max } \leq 1$, and $0 \leq u_{3 \min } \leq u_{3}(t) \leq$ $\left.u_{3 \max } \leq 1,0 \leq u_{4 \min } \leq u_{4}(t) \leq u_{4 \max } \leq 1 / t \in\left[0, T_{f}\right]\right\}$ is convex and closed by definition

(iv) All the right-hand sides of equations of system are continuous, bounded above by a sum of bounded control and state, and can be written as a linear function of $u, v$, and $w$ with coefficients depending on the time and state

(v) The integrand in the objective functional, $C_{T}(t)-$ $D(t)-E(t)+(A / 2) u_{1}^{2}(t)+(B / 2) u_{2}^{2}(t)+(F / 2) u_{3}^{2}(t)$ $+(G / 2) u_{4}^{2}(t)$, is clearly convex on $U$

(vi) It rests to show that there exist constants $\zeta_{1}, \zeta_{2}$, $\zeta_{3}, \zeta_{4}, \zeta_{5}>0$ and $\zeta$ such that $C_{T}(t)-D(t)-E(t)+$ $(A / 2) u_{1}^{2}(t)+(B / 2) u_{2}^{2}(t)+(F / 2) u_{3}^{2}(t)+(G / 2) u_{4}^{2}(t)$ satisfies

$$
\begin{gathered}
C_{T}(t)-D(t)-E(t)+\frac{A}{2} u_{1}^{2}(t)+\frac{B}{2} u_{2}^{2}(t)+\frac{F}{2} u_{3}^{2}(t)+\frac{G}{2} u_{4}^{2}(t) \\
\geq-\zeta_{1}+\zeta_{2}\left|u_{1}\right|^{\zeta}+\zeta_{3}\left|u_{2}\right|^{\zeta}+\zeta_{4}\left|u_{3}\right|^{\zeta}+\zeta_{5}\left|u_{4}\right|^{\zeta}
\end{gathered}
$$

The state variables are being bounded; let $\zeta_{1}=\sup _{t \in\left[0, T_{f}\right]}\left(C_{T}(t)\right.$ $-D(t)-E(t)), \zeta_{2}=A, \zeta_{3}=B, \zeta_{4}=F, \zeta_{5}=G$ and $\zeta=2$; then, it follows that

$$
\begin{gathered}
C_{T}(t)-D(t)-E(t)+\frac{A}{2} u_{1}^{2}(t)+\frac{B}{2} u_{2}^{2}(t)+\frac{F}{2} u_{3}^{2}(t)+\frac{G}{2} u_{4}^{2}(t) \\
\geq-\zeta_{1}+\zeta_{2}\left|u_{1}\right|^{\zeta}+\zeta_{3}\left|u_{2}\right|^{\zeta}+\zeta_{4}\left|u_{3}\right|^{\zeta}+\zeta_{5}\left|u_{4}\right|^{\zeta} .
\end{gathered}
$$

Then, from Fleming and Rishel [23], we conclude that there exists an optimal control.

4.2. Characterization of the Optimal Control. In order to derive the necessary conditions for the optimal control, we apply Pontryagin's maximum principle to the Hamiltonian $\widehat{H}$ at time $t$ defined by

$$
\begin{aligned}
\widehat{H}(t)= & C_{T}(t)-D(t)-E(t)+\frac{A}{2} u_{1}^{2}(t)+\frac{B}{2} u_{2}^{2}(t)+\frac{F}{2} u_{3}^{2}(t) \\
& +\frac{G}{2} u_{4}^{2}(t)+\sum_{i=1}^{6} \lambda_{i}(t) f_{i}\left(H, P, E, D, \mathbf{C}_{T}, \mathbf{C}_{S}\right)
\end{aligned}
$$

where $f_{i}$ is the right side of the difference equation of the $i^{\text {th }}$ state variable.

Theorem 5. Given the optimal controls $\left(u_{1}^{*}, u_{2}^{*}, u_{3}^{*}, u_{4}^{*}\right)$ and the solutions $H^{*}, P^{*}, E^{*}, D^{*}, C_{T}^{*}$, and $C_{S}^{*}$ of the corresponding state system (18), there exists adjoint variables $\lambda_{1}, \lambda_{2}, \lambda_{3}, \lambda_{4}$, $\lambda_{5}$, and $\lambda_{6}$ satisfying

$$
\begin{aligned}
\lambda_{1}^{\prime}= & \lambda_{1}\left(\mu+\theta_{1}+\theta_{2}\right)-\lambda_{2} \theta_{1}-\lambda_{3} \theta_{2}, \\
\lambda_{2}^{\prime}= & \lambda_{2}\left(\mu+\beta_{1}+\beta_{3}\right)-\lambda_{4} \beta_{1}-\lambda_{5} \beta_{3}, \\
\lambda_{3}^{\prime}=1 & +\lambda_{3}\left[\mu+\gamma\left(1-u_{4}(t)\right)\right] \\
& -\lambda_{4}\left[\gamma\left(1-u_{4}(t)\right)-\alpha_{1}\left(1-u_{2}(t)\right) \frac{D(t)}{N}\right] \\
& -\lambda_{5}\left[\alpha_{1}\left(1-u_{2}(t)\right) \frac{D(t)}{N}-\alpha_{2}\left(1-u_{3}(t)\right) \frac{\mathbf{C}_{T}(t)}{N}\right] \\
& -\lambda_{6}\left[\alpha_{2}\left(1-u_{3}(t)\right) \frac{\mathbf{C}_{T}(t)}{N}\right], \\
\lambda_{4}^{\prime}=1 & -\lambda_{4}\left[\alpha_{1}\left(1-u_{2}(t)\right) \frac{E(t)}{N}+\left(\mu+\beta_{2}+\eta_{2}\right)\right] \\
& -\lambda_{5}\left[\beta_{2}+\alpha_{1}\left(1-u_{2}(t)\right) \frac{E(t)}{N}\right]-\lambda_{6} \eta_{2}, \\
\lambda_{5}^{\prime}= & -1-\lambda_{4} u_{1}(t)+\lambda_{5}\left[-\alpha_{2}\left(1-u_{3}(t)\right) \frac{E(t)}{N}-\left(\mu+\eta_{1}\right.\right. \\
& \left.\left.+u_{1}(t)\right)\right]-\lambda_{6}\left[\eta_{1}+\alpha_{2}\left(1-u_{3}(t)\right) \frac{E(t)}{N}\right],
\end{aligned}
$$

$\lambda_{6}^{\prime}=\lambda_{6}(\mu+\delta)$

With the transversality conditions at time $T_{f}, \lambda_{1}\left(T_{f}\right)$ $=0, \lambda_{2}\left(T_{f}\right)=0, \lambda_{3}\left(T_{f}\right)=1, \lambda_{4}\left(T_{f}\right)=1, \lambda_{5}\left(T_{f}\right)=-1$, and $\lambda_{6}$ $\left(T_{f}\right)=0$.

Furthermore, for $t \in\left[0, T_{f}\right]$, the optimal controls $u_{1}^{*}, u_{2}^{*}$, $u_{3}^{*}$, and $u_{4}^{*}$ are given by

$$
\begin{aligned}
& u_{1}^{*}=\min \left(1, \max \left(0, \frac{\left(\lambda_{5}-\lambda_{4}\right)}{A} \mathbf{C}_{T}(t)\right)\right), \\
& u_{2}^{*}=\min \left(1, \max \left(0, \alpha_{1} \times \frac{\left(\lambda_{5}-\lambda_{4}\right)}{B} \times \frac{D(t) E(t)}{N}\right)\right), \\
& u_{3}^{*}=\min \left(1, \max \left(0, \alpha_{2} \times \frac{\left(\lambda_{6}-\lambda_{5}\right)}{F} \times \frac{\mathbf{C}_{T}(t) E(t)}{N}\right)\right), \\
& u_{4}^{*}=\min \left(1, \max \left(0, \frac{\left(\lambda_{4}-\lambda_{3}\right)}{G} \times \gamma E(t)\right)\right),
\end{aligned}
$$




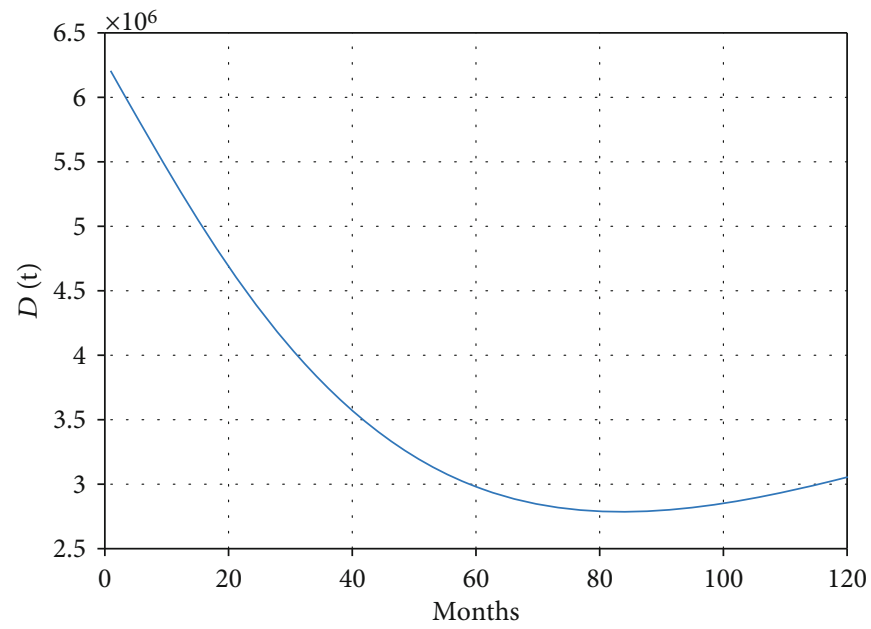

_ Diabetics without complications

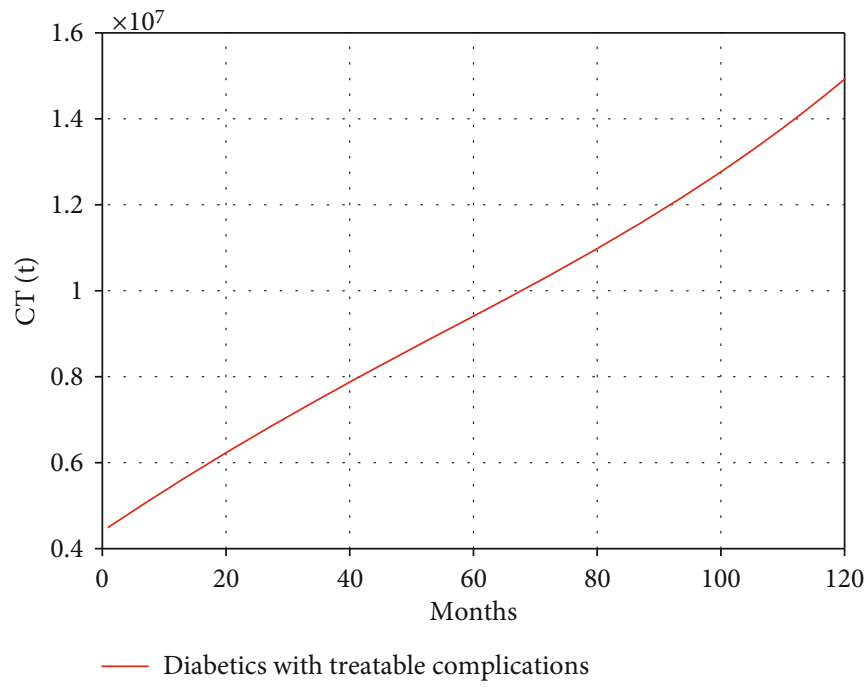

- Diabetics with treatable complications

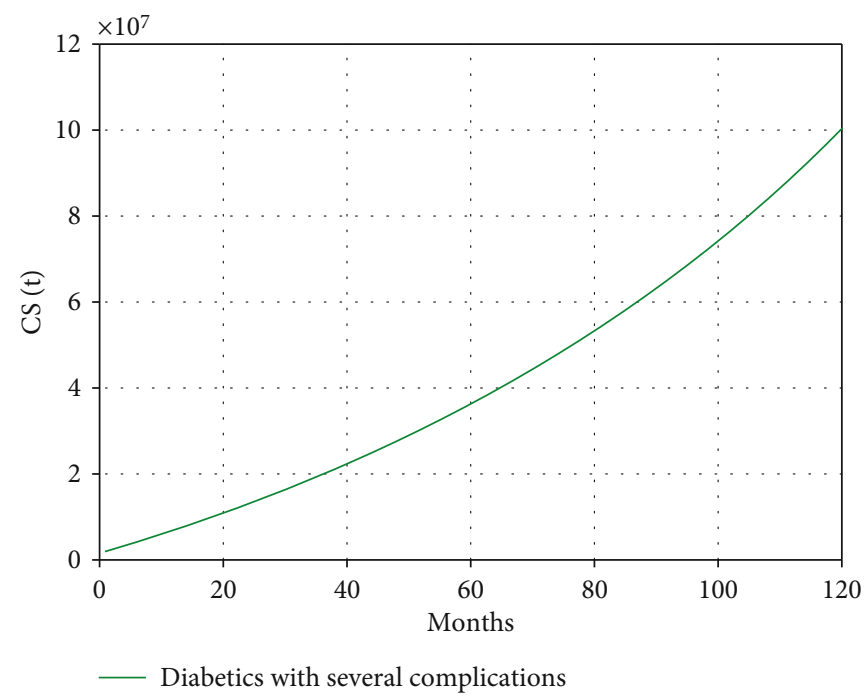

FIgURE 2: The evolution of the number of diabetics with and without complications without controls.

Proof. The Hamiltonian is defined as follows:

$$
\begin{aligned}
\widehat{H}(t)= & C_{T}(t)-D(t)-E(t)+\frac{A}{2} u_{1}^{2}(t)+\frac{B}{2} u_{2}^{2}(t)+\frac{F}{2} u_{3}^{2}(t) \\
& +\frac{G}{2} u_{4}^{2}(t)+\sum_{i=1}^{6} \lambda_{i}(t) f_{i}\left(H, P, E, D, \mathbf{C}_{T}, \mathbf{C}_{S}\right),
\end{aligned}
$$

where

$$
\begin{gathered}
f_{1}\left(H, P, E, D, \mathbf{C}_{T}, \mathbf{C}_{S}\right)=I-\left(\mu+\theta_{1}+\theta_{2}\right) H(t) \\
f_{2}\left(H, P, E, D, \mathbf{C}_{T}, \mathbf{C}_{S}\right)=\theta_{1} H(t)-\left(\mu+\beta_{1}+\beta_{3}\right) P(t), \\
f_{3}\left(H, P, E, D, \mathbf{C}_{T}, \mathbf{C}_{S}\right)=\theta_{2} H(t)-\mu E(t)+\gamma\left(1-u_{4}(t)\right) \\
E(t), \\
\quad f_{4}\left(H, P, E, D, \mathbf{C}_{T}, \mathbf{C}_{S}\right)=\beta_{1} P(t)+\gamma\left(1-u_{4}(t)\right) E(t)-\alpha_{1}(1 \\
\left.-u_{2}(t)\right)(D(t) E(t) / N)-\left(\mu+\beta_{2}+\eta_{2}\right) D(t)+u_{1}(t) C_{T}(t), \\
f_{5}\left(H, P, E, D, \mathbf{C}_{T}, \mathbf{C}_{S}\right)=\beta_{3} P(t)+\beta_{2} D(t)+\alpha_{1}\left(1-u_{2}(t)\right) \\
(D(t) E(t) / N)-\alpha_{2}\left(1-u_{3}(t)\right)\left(\mathbf{C}_{T}(t) E(t) / N\right)-\left(\mu+\eta_{1}\right) C_{T}(t) \\
-u_{1}(t) C_{T}(t), \\
\quad f_{6}\left(H, P, E, D, \mathbf{C}_{T}, \mathbf{C}_{S}\right)=\eta_{2} D(t)+\eta_{1} \mathbf{C}_{T}(t)+\alpha_{2}\left(1-u_{3}(t)\right) \\
\left(\mathbf{C}_{T}(t) E(t) / N\right)-(\mu+\delta) C_{S}(t) .
\end{gathered}
$$

For $t \in\left[0, T_{f}\right]$, the adjoint equations and transversality conditions can be obtained by using Pontryagin's maximum principle $[13,25]$ such that

$$
\begin{aligned}
\lambda_{1}^{\prime}= & -\frac{\partial \widehat{H}}{\partial H}=\lambda_{1}\left(\mu+\theta_{1}+\theta_{2}\right)-\lambda_{2} \theta_{1}-\lambda_{3} \theta_{2}, \\
\lambda_{2}^{\prime}= & -\frac{\partial \widehat{H}}{\partial P}=\lambda_{2}\left(\mu+\beta_{1}+\beta_{3}\right)-\lambda_{4} \beta_{1}-\lambda_{5} \beta_{3}, \\
\lambda_{3}^{\prime}= & -\frac{\partial \widehat{H}}{\partial E}=1+\lambda_{3}\left[\mu+\gamma\left(1-u_{4}(t)\right)\right] \\
& -\lambda_{4}\left[\gamma\left(1-u_{4}(t)\right)-\alpha_{1}\left(1-u_{2}(t)\right) \frac{D(t)}{N}\right] \\
& -\lambda_{5}\left[\alpha_{1}\left(1-u_{2}(t)\right) \frac{D(t)}{N}-\alpha_{2}\left(1-u_{3}(t)\right) \frac{\mathbf{C}_{T}(t)}{N}\right] \\
& -\lambda_{6}\left[\alpha_{2}\left(1-u_{3}(t)\right) \frac{\mathbf{C}_{T}(t)}{N}\right]
\end{aligned}
$$


TABLE 1: Parameter values used in numerical simulation.

\begin{tabular}{lcc}
\hline Paramater & Value in $\mathrm{mth}^{-1}$ & Description \\
\hline$\mu$ & $0: 02$ & Natural mortality \\
$\delta$ & $0: 001$ & $\begin{array}{c}\text { Mortality rate due to complications } \\
\beta_{1}\end{array}$ \\
$\beta_{2}$ & $0: 2$ & $\begin{array}{c}\text { The probability of developing diabetes } \\
\beta_{3}\end{array}$ \\
$a_{1}$ & 0.08 & The probability of a diabetic person developing a complication \\
$Y$ & $0: 4$ & The rate of negative impact on diabetics without complications \\
$I$ & 0.06 & Rate of patients become diabetic without complications through lifestyle \\
$a_{2}$ & 2000000 & The rate of negative impact on diabetics with treatable complications \\
$\theta_{1}$ & $0: 6$ & Rate of prediabetic people through genetic factor \\
$\theta_{1}$ & $0: 1$ & Rate of prediabetic people through lifestyle factor \\
$\eta_{1}$ & $0: 2$ & The probability of a diabetic person developing a serious complication \\
$\eta_{2}$ & $0: 6$ & Rate of diabetics whose serious complications are because of a sudden shock
\end{tabular}

TABLE 2: Evolution of number of diabetics without control after 120 days.

\begin{tabular}{lc}
\hline Population without control after 120 days & Without control \\
\hline Diabetics without complications & $3: 05 \times 10^{6}$ \\
Diabetics with treatable complications & $1: 49 \times 10^{7}$ \\
Diabetics with serious complications & $1 \times 10^{8}$ \\
\hline
\end{tabular}

$$
\begin{aligned}
\lambda_{4}^{\prime}= & -\frac{\partial \widehat{H}}{\partial D}=1-\lambda_{4}\left[\alpha_{1}\left(1-u_{2}(t)\right) \frac{E(t)}{N}+\left(\mu+\beta_{2}+\eta_{2}\right)\right] \\
& -\lambda_{5}\left[\beta_{2}+\alpha_{1}\left(1-u_{2}(t)\right) \frac{E(t)}{N}\right]-\lambda_{6} \eta_{2}, \\
\lambda_{5}^{\prime}= & -\frac{\partial \widehat{H}}{\partial \mathbf{C}_{T}}=-1-\lambda_{4} u_{1}(t)+\lambda_{5}\left[-\alpha_{2}\left(1-u_{3}(t)\right) \frac{E(t)}{N}\right. \\
& \left.-\left(\mu+\eta_{1}+u_{1}(t)\right)\right]-\lambda_{6}\left[\eta_{1}+\alpha_{2}\left(1-u_{3}(t)\right) \frac{E(t)}{N}\right], \\
\lambda_{6}^{\prime}= & -\frac{\partial \widehat{H}}{\partial \mathbf{C}_{S}}=\lambda_{6}(\mu+\delta) .
\end{aligned}
$$

For, $t \in\left[0, T_{f}\right]$ the optimal controls $u_{1}^{*}, u_{2}^{*}, u_{3}^{*}$, and $u_{4}^{*}$ can be solved from the optimality condition,

$$
\begin{aligned}
& \frac{\partial \widehat{H}}{\partial u_{1}}=0, \\
& \frac{\partial H}{\partial u_{2}}=0, \\
& \frac{\partial \widehat{H}}{\partial u_{3}}=0, \\
& \frac{\partial \widehat{H}}{\partial u_{4}}=0,
\end{aligned}
$$

that are

$$
\begin{aligned}
& -\frac{\partial \widehat{H}}{\partial u_{1}}=-A u_{1}(t)+\left(\lambda_{2}-\lambda_{4}\right) \mathbf{C}_{T}(t)=0, \\
& -\frac{\partial \widehat{H}}{\partial u_{2}}=-B u_{2}(t)+\alpha_{1}\left(\lambda_{5}-\lambda_{4}\right) \frac{D(t) E(t)}{N}=0, \\
& -\frac{\partial \widehat{H}}{\partial u_{3}}=-F u_{3}(t)+\alpha_{2}\left(\lambda_{6}-\lambda_{5}\right) \frac{\mathbf{C}_{T}(t) E(t)}{N}=0, \\
& -\frac{\partial \widehat{H}}{\partial u_{4}}=-G u_{4}(t)+\left(\lambda_{4}-\lambda_{3}\right) \gamma E(t)=0 .
\end{aligned}
$$

We have

$$
\begin{aligned}
& u_{1}(t)=\frac{\left(\lambda_{2}-\lambda_{4}\right)}{A} \mathbf{C}_{T}(t), \\
& u_{2}(t)=\alpha_{1} \times\left(\left(\lambda_{5}-\lambda_{4}\right) / B\right) \times(D(t) E(t) / N), \\
& u_{3}(t)=\alpha_{2} \times \frac{\left(\lambda_{6}-\lambda_{5}\right)}{F} \times \frac{\mathbf{C}_{T}(t) E(t)}{N}, \\
& u_{4}(t)=\frac{\left(\lambda_{4}-\lambda_{3}\right)}{G} \times \gamma E(t) .
\end{aligned}
$$

By the bounds in $U$ of the controls, it is easy to obtain $u_{1}^{*}, u_{2}^{*}, u_{3}^{*}$, and $u_{4}^{*}$ and are given in (13-16) the form of system and in the form of system (18).

\section{Numerical Simulation}

In this section, we present the results obtained by solving numerically the optimality system (18). In our control problem, we have initial conditions for the state variables and terminal conditions for the adjoints. That is, the optimality 

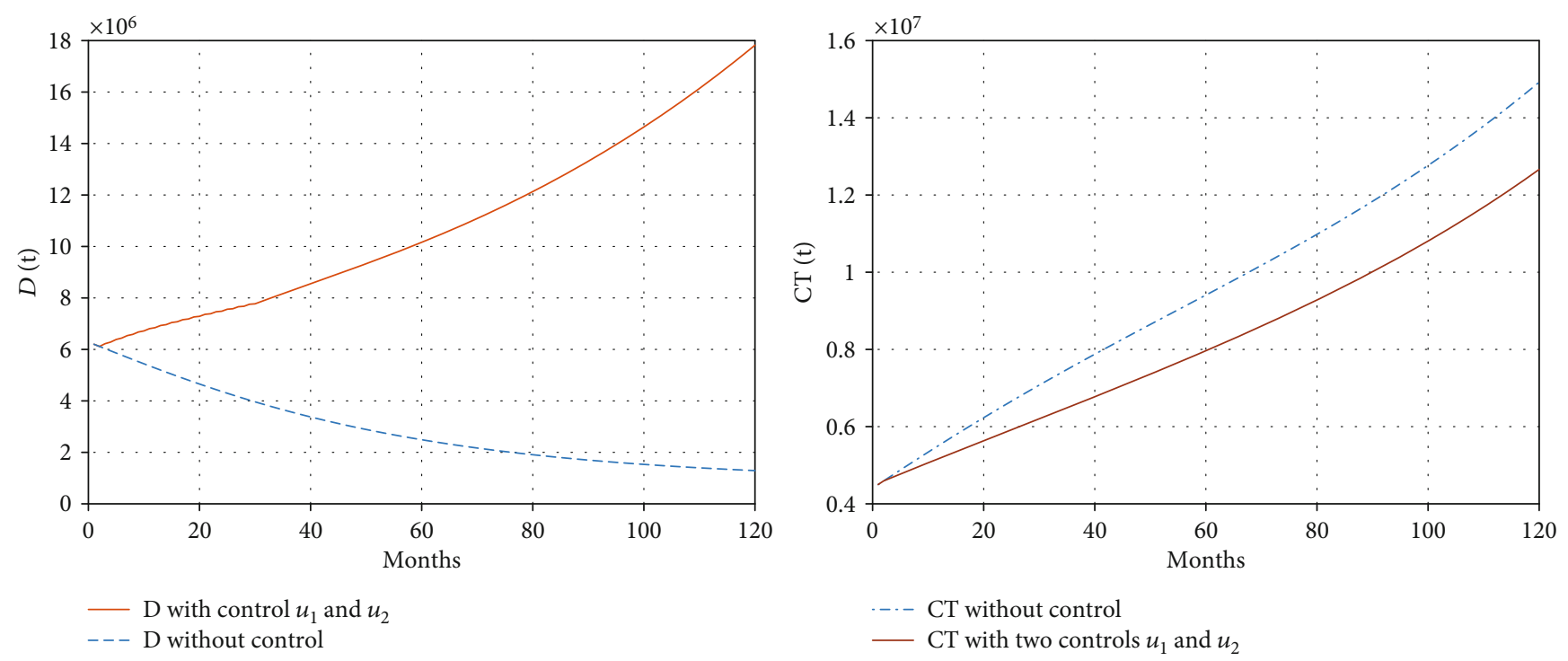

Figure 3: The evolution of the number of diabetics with and without complications without controls.

TABLE 3: Evolution of the number of diabetics with two controls $u_{1}(t)$ and $u_{2}(t)$ after 120 days.

\begin{tabular}{lcc}
\hline $\begin{array}{l}\text { Population without control after } \\
120 \text { days }\end{array}$ & $\begin{array}{c}\text { Without } \\
\text { control }\end{array}$ & $\begin{array}{c}\text { With two controls } \\
u_{1}(t) \text { and } u_{2}(t)\end{array}$ \\
\hline $\begin{array}{l}\text { Diabetics without complications } \\
\text { Diabetics with treatable }\end{array}$ & $10^{7}$ & $26 \times 10^{6}$ \\
complications & & $2.14 \times 10^{7}$ \\
\hline
\end{tabular}

system is a two-point boundary value problem with separated boundary conditions at times step $i=0$ and $i=T_{f}$. We solve the optimality system by an iterative method with forward solving of the state system followed by backward solving of the adjoint system. We start with an initial guess for the controls at the first iteration, and then before the next iteration, we update the controls by using the characterization. We continue until convergence of successive iterates is achieved. A code is written and compiled in MATLAB using the following data.

Different simulations can be carried out using various values of parameters. In the present numerical approach, we use the following parameters values taken from [6].

Since control and state functions are on different scales, the weight constant value is chosen as follows: $A=10000$, $B=10000, F=10000$, and $G=10000$ and with the intial value of $H(0)=14000000, P(0)=6660000, E(0)=13000000, D(0)$ $=6200000, C_{T}(0)=4500000$, and $C_{S}(0)=2000000$ (Figure 2).

After the parameter values (Tables 1 and 2), we noted that diabetics without complications after 120 months decreased from $6.2 \times 10^{6}$ to $3.05 \times 10^{6}$ (Figure 2) This transformation is due to three main things: first, it is by the genetic factors. Second, due to the negative impact of behavioral factors on the patient (nutrition pattern and psychological and moral problems) and the third by sudden shock (family problem, work problem), we noted that diabetics with treatable complications are increasing. Indeed, we noted that the number of the transition becomes from $4.5 \times 10^{6}$ to $1.49 \times 10^{7}$ (Figure 2) and, as mentioned above, has disease progression for diabetics without complications, and also a sudden shift in the potential for people diagnosed with diabetes by means of genetics and with negative impact of behavioral factors.

We noted that diabetics with serious complications are increasing and that the number of the transition becomes from $2 \times 10^{6}$ to $1 \times 10^{8}$ (Figure 1) and, as mentioned above, has disease progression for diabetics without complications by sudden shock and with negative impact of behavioral factors, and by developing the disease of diabetics with treatable complications.

In this formulation, there are initial conditions for the state variables and terminal conditions for the adjoints.

That is, the optimality system is a two-point boundary value problem with separated boundary conditions at time steps $i=0$ and $i=T$. We solve the optimality system by an iterative method with forward solving of the state system followed by backward solving of the adjoint system. We start with an initial guess for the controls at the first iteration, and then before the next iteration, we update the controls by using the characterization.

We continue until convergence of successive iterates is achieved.

The proposed control strategy in this work helps to achieve several objectives.

5.1. Strategy $A$. In this strategy, we applied two controls $u_{1}(t)$ and $u_{2}(t)$ in order to reduced the number of diabetics with treatable complications to diabetics without complications, through Figure 2, we noted that after applied different strategics, the number of diabetics with treatable complications decreased from $1.49 \times 10^{7}$ to $1.26 \times 10^{7}$ by the end of the strategy (Figure 3).

The reason of this increase was justified by the fact that the number of diabetics with treatable complications will become diabetics without complications. For improving the 

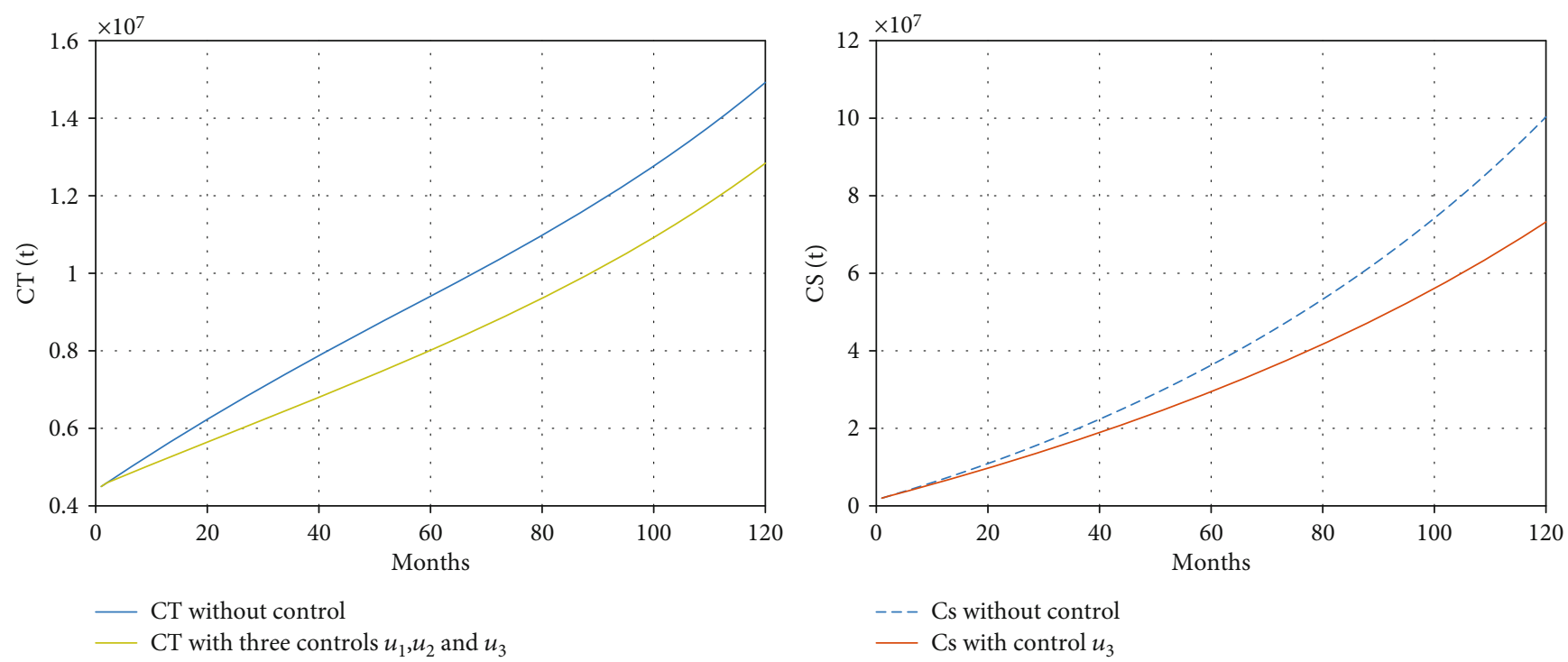

FIgURE 4: The evolution of the number of diabetics with treatable and serious complications with three controls $u_{1}(t), u_{2}(t)$, and $u_{3}(t)$.

TABLE 4: Evolution of number of diabetics with three controls $u_{1}(t), u_{2}(t)$, and $u_{3}(t)$ after 120 days.

\begin{tabular}{lcc}
\hline $\begin{array}{l}\text { Population without control } \\
\text { after } 120 \text { days }\end{array}$ & $\begin{array}{c}\text { Without } \\
\text { control }\end{array}$ & $\begin{array}{c}\text { With three controls } \\
u_{1}(t), u_{2}(t), \text { and } u_{3}(t)\end{array}$ \\
\hline $\begin{array}{l}\text { Diabetics with treatable } \\
\text { complications }\end{array}$ & $1: 5 \times 10^{7}$ & $1.28 \times 10^{7}$ \\
$\begin{array}{l}\text { Diabetics with serious } \\
\text { complications }\end{array}$ & $10^{8}$ & $7: 32 \times 10^{7}$ \\
\hline
\end{tabular}

effectiveness of this strategy, we added the elements of follow-up and psychological support and education about the negative impact of behavioral factors which are represented in the proposed strategy by the optimal controls variables $u_{1}(t)$ and $u_{2}(t)$ (Figure 3 and Table 3 ), combining follow-up and psychological support with treatment and education results in an obvious decreased in the number of diabetics with treatable complications.

\subsection{Strategy C: Control with Awareness Program,Treatment,} and Psychological Support with Follow-Up. We combined three optimal controls $u_{1}(t), u_{2}(t)$, and $u_{3}(t)$.

In this strategy, the three optimal controls $u_{1}(t), u_{2}(t)$, and $u_{3}(t)$ are activated at the same time, in order to reduced the number of diabetics with treatable complications to diabetics without complications (Figure 4).

In this strategy (Figure 4 and Table 4 ), we used three controls optimal $u_{1}(t), u_{2}(t)$, and $u_{3}(t)$. That is, we combined the previous two strategies to achieved better results that represented treatment, and psychological support with followup, and also awareness program through education and media for lower the negative impact of behavioral factors. In Figure 4 and Table 4, we observe that the number of diabetics with treatable complications is decreasing from $1.5 \times$
$10^{7}$ to $1.28 \times 10^{7}$, and also, the number of diabetics with serious complications is decreasing from $10^{8}$ to $7.32 \times 10^{7}$.

5.3. Strategy D: Prevention and Protection E from Diabetes. We we use only the optimal control $u_{4}(t)$.

In this strategy, we focus the effort of the awareness campaign to reduce the negative impact of behavioral factors (Figure 5).

In this strategy, we used control $u_{4}(t)$ (Figure 5 and Table 5); the objective of this control $u_{4}(t)$ is to raise awareness campaigns for this target group on the risks of diabetes and its complications as cardiovascular disease, blindness, kidney failure, and lower limb amputation, with tracking healthy and balanced diet program. after Figure 5 and Table 5, we observed that the number of diabetics without complications is decreasing from $1.29 \times 10^{6}$ to $10^{6}$.

Remark 6. We could also merge multiple assemblies as $\left(u_{1}(t), u_{2}(t), u_{3}(t)\right)$, and $\left(u_{1}(t), u_{2}(t), u_{3}(t), u_{4}(t)\right)$ and thus get a variety of results.

\section{Conclusion}

In this paper, we formulated a mathematical model of populations of diabetics, having six compartments: prediabetics through the genetics effects and others by behavioral factors, diabetics without complications, and diabetics with treatable and serious complications, in order to minimize the number of diabetics with treatable complications, and reduce the effect of behavioral factors. We also introduced four controls which, respectively, represent awareness program through education and media, treatment, and psychological support with follow-up. We applied the results of the control theory, and we managed to obtain the characterizations of the optimal controls. The numerical simulation of the obtained results showed the effectiveness of the proposed control strategies. 

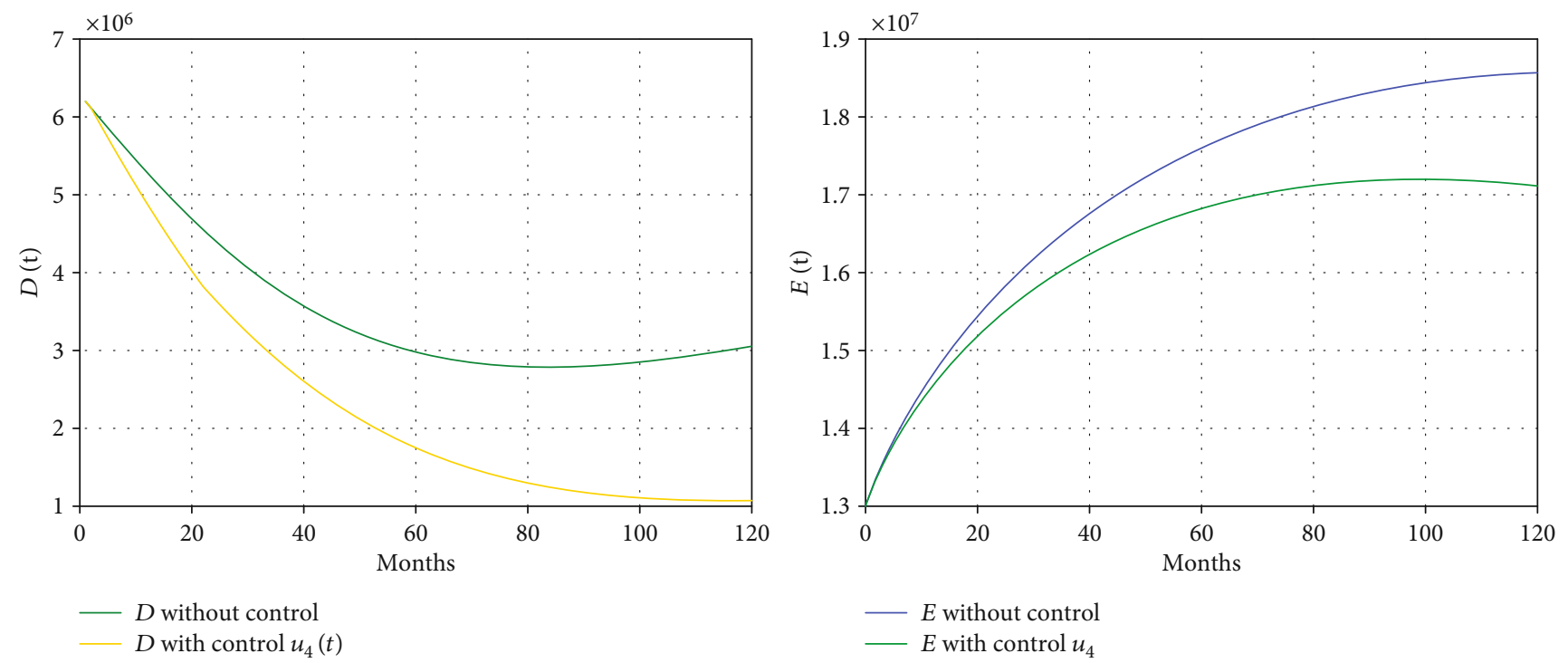

FIGURE 5: The evolution of the number of diabetics without complications with control $u_{4}(t)$.

TABLE 5: Evolution of number of diabetics with control $u_{4}(t)$ after 120 days.

\begin{tabular}{lcc}
\hline $\begin{array}{l}\text { Population without control after } \\
120 \text { days }\end{array}$ & $\begin{array}{c}\text { Without } \\
\text { control }\end{array}$ & $\begin{array}{c}\text { With control } \\
u_{4}(t)\end{array}$ \\
\hline $\begin{array}{l}\text { Diabetics without complications } \\
\begin{array}{l}\text { Prediabetics due to effect of } \\
\text { behavioral factors }\end{array}\end{array}$ & $1: 29 \times 10^{6}$ & $10^{6}$ \\
\hline
\end{tabular}

\section{Data Availability}

The disciplinary data used to support the Öndings of this study have been deposited in the Network Repository (http://www.networkrepository.com).

\section{Conflicts of Interest}

The authors declare that they have no conflicts of interest.

\section{Acknowledgments}

The authors would like to thank Dr. Bruno Carpentieri and all the members of the Editorial Board who were responsible for dealing with this paper, and the anonymous referees for their valuable comments and suggestions, improving the content of this paper.

\section{References}

[1] World Health Organisation, Definition and diagnosis of diabetes mellitus and intermediate hyperglycemia, WHO, Geneva, 2016.

[2] The American Diabetes Association (ADA), How to Prevent and Treat PrediabetesMarch 2018, http://www.diabetesforecast .org/2018/02-mar-apr/how-to-prevent-and-treat.html.

[3] International Diabetes Federation (IDF), IDF DIABETES ATLAS, Ninth editionNinth edition, , 2019.
[4] A. Boutayeb and A. Chetouani, "A population model of diabetes and prediabetes," International Journal of Computer Mathematics, vol. 84, no. 1, pp. 57-66, 2007.

[5] M. Derouich, A. Boutayeb, W. Boutayeb, and M. Lamlili, "Optimal control approach to the dynamics of a population of diabetics," Applied Mathematical Sciences, vol. 8, no. 56, pp. 2773-2782, 2014.

[6] A. Kouidere, O. Balatif, H. Ferjouchia, A. Boutayeb, and M. Rachik, "Optimal control strategy for a discrete time to the dynamics of a population of diabetics with highlighting the impact of living environment," Discrete Dynamics in Nature and Society, vol. 2019, Article ID 6342169, 8 pages, 2019.

[7] O. Balatif, I. Abdelbaki, M. Rachik, and Z. Rachik, "Optimal control for multi-input bilinear systems with an application in cancer chemotherapy," International Journal of Scientific and Innovative Mathematical Research (IJSIMR), vol. 3, no. 2, pp. 22-31, 2015.

[8] W. Boutayeb, M. E. N. Lamlili, A. Boutayeb, and M. Derouich, "A simulation model for the dynamics of a population of diabetics with and without complications using optimal control," Lecture Notes in Computer Science (including subseries Lecture Notes in Artificial Intelligence and Lecture Notes in Bioinformatics), vol. 9043, pp. 589-598, 2015.

[9] A. H. Permatasari, R. H. Tjahjana, and T. Udjiani, "Existence and characterization of optimal control in mathematics model of diabetics population," Journal of Physics: Conference Series, vol. 983, p. 012069, 2018.

[10] P. Widyaningsih, R. C. Affan, and D. R. S. Saputro, "A mathematical model for the epidemiology of diabetes mellitus with lifestyle and genetic factors," Journal of Physics: Conference Series, vol. 1028, p. 012110, 2018.

[11] A. Kouidere, A. Labzai, B. Khajji et al., "Optimal control strategy with multi-delay in state and control variables of a discrete mathematical modeling for the dynamics of diabetic population," Communications in Mathematical Biology and Neuroscience, vol. 2020, p. 14, 2020.

[12] A. De Gaetano and T. A. Hardy, "A novel fast-slow model of diabetes progression: insights into mechanisms of response 
to the interventions in the diabetes prevention program," PLoS One, vol. 14, no. 10, article e0222833, 2019.

[13] B. Omar, K. Bouchaib, R. Mostafa, and E. Benlahmar, "Optimal Control of an SIR Model with Delay in State and Control Variables," ISRN Biomathematics, vol. 2013, Article ID 403549, 7 pages, 2013.

[14] H. Laarabi, A. Abta, and K. Hattaf, "Optimal control of a delayed SIRS epidemic model with vaccination and treatment," Acta Biotheoretica, vol. 63, no. 2, pp. 87-97, 2015.

[15] A. Barbagallo and M. A. Ragusa, "On Lagrange duality theory for dynamics vaccination games," Ricerche di Matematica, vol. 67, no. 2, pp. 969-982, 2018.

[16] A. Labzai, O. Balatif, and M. Rachik, "Optimal control strategy for a discrete time smoking model with specific saturated incidence rate," Discrete Dynamics in Nature and Society, vol. 2018, Article ID 5949303, 10 pages, 2018.

[17] A. El Bhih, Y. Benfatah, S. Ben Rhila, M. Rachik, and A. El Alami Laaroussi, "A spatiotemporal prey-predator discrete model and optimal controls for environmental sustainability in the multifishing areas of Morocco," Discrete dynamics in nature and society, vol. 2020, Article ID 2780651, 18 pages, 2020.

[18] A. El Bhih, R. Ghazzali, S. Ben Rhila, M. Rachik, and A. El Alami Laaroussi, "A discrete mathematical Modeling and optimal control of the rumor propagation in online social network," Discrete dynamics in nature and society, vol. 2020, Article ID 4386476, 12 pages, 2020.

[19] A. Kouidere, B. Khajji, A. El Bhih, O. Balatif, and M. Rachik, "A mathematical modeling with optimal control strategy of transmission of COVID-19 pandemic virus," Communications in Mathematical Biology and Neuroscience, vol. 2020, article 24, 2020.

[20] A. B. Gumel, P. N. Shivakumar, and B. M. Sahai, "A mathematical model for the dynamics of HIV-1 during the typical course of infection," Proceeding of the 3rd World Congress of Nonlinear Analysts, vol. 47, pp. 2073-2083, 2011.

[21] B. Khajji, A. Labzai, A. Kouidere, O. Balatif, and M. Rachik, “A discrete mathematical modeling of the influence of alcohol treatment centers on the drinking dynamics using optimal control," Journal of Applied Mathematics, vol. 2020, Article ID 9284698, 13 pages, 2020.

[22] G. Birkho and G. C. Rota, Ordinary differential equations, John Wiley \& Sons, New York, 4 edition, 1989.

[23] W. H. Fleming and R. W. Rishel, Deterministic and Stochastic Optimal Control, Springer, New York, NY, USA, 1975.

[24] W. E. Boyce, D. P. RC, and H. Villagómez Velázquez, "Elementary Differential Equations and Boundary Value Problems Chapter 7," in Systems of first order linear equations (Theorem 7.1.1) Page 362, John Wiley \& Sons, New York, 2009.

[25] L. S. Pontryagin, V. G. Boltyanskii, R. V. Gamkrelidze, and E. F. Mishchenko, The Mathematical Theory of Optimal Processes, John Wiley \& Sons, London, UK, 1962. 